\title{
Phase resolved optical polarization of the Crab pulsar
}

\section{Agnieszka Słowikowska*}

IESL, Foundation for Research and Technology - Hellas, P.O. Box 1385, 71110 Heraklion,

Crete, Greece

E-mail: aga@physics.uoc.gr

\section{Gottfried Kanbach}

MPI für extraterr. Physik, Postfach 1312, 85741 Garching, Germany

E-mail: gok@mpe.mpg.de

\section{Alexander Stefanescu}

MPI für extraterr. Physik, Postfach 1312, 85741 Garching, Germany

E-mail: astefan@mpe.mpg.de

\begin{abstract}
The linear polarization of the Crab pulsar and its close environment was derived from observations with the high-speed photo-polarimeter OPTIMA at the $2.56 \mathrm{~m}$ Nordic Optical Telescope in the optical spectral range $(400-750 \mathrm{~nm})$. The high time resolution and photon statistic allows us to identify very fine features in the degree and angle of linear polarization as a function of pulsar rotational phase never achieved before. The optical data show surprising correlations with the phase structure at radio frequency. Our observations show that there exists a subtle connection between presumed non-coherent (optical) and coherent (radio) emissions. This finding supports previously detected correlations between the optical intensity of the Crab and the occurrence of giant radio pulses. Interpretation of our observations require more elaborate theoretical models than those currently available in the literature.
\end{abstract}

Polarimetry days in Rome: Crab status, theory and prospects October 16-17 2008

Rome, Italy

\footnotetext{
* Speaker.
} 


\section{Introduction}

Pulsars - fast rotating, highly magnetized neutron stars - are very faint objects in the optical range of the electromagnetic spectrum. Yet, despite the increasing number of optical pulsars (fourteen are known), only for five of them an attempt to get their and/or their nebulae polarization characteristics was made (for summary see [1]). However, only for the brightest, i.e. the Crab pulsar $\left(m_{V}=16.6\right)$, fully phase resolved polarization measurements have been possible so far. Precise measurements of polarization degree (PD) and position angle (PA) as a function of pulse phase in a wide range of energy domains would provide deeper insight into the details of the pulsars emission mechanisms. However, since fast X- and $\gamma$-ray polarimetry is unavailable so far, we are restricted to the optical domain.

\section{The OPTIMA instrument}

The primary science goal for Optical Pulsar TIMing Analyzer (OPTIMA, [河]) is to detect and measure the optical light curves of young high-energy pulsars known to emit $\mathrm{X}$ - and $\gamma$-photons. Another class of targets for a fast photometer are compact objects in binary systems (white dwarfs, neutron stars, black hole candidates) and their environment. In these sources quasi-periodic, as well as, irregular and random intensity fluctuations are expected. This calls for the detection and timing of single photons in order to be able to derive light curves with any suitable binning after the observations. The absolute arrival time of individual photons is also needed for the correlation of OPTIMA data with measurements from other ground or space observatories. The timing of individual photons is controlled by signals from the Global Positioning System (GPS) to an absolute accuracy of $\sim 2 \mu \mathrm{s}$, although the readout system limits the resolution to $\sim 4 \mu \mathrm{s}$.

To minimize the dilution of the source's signal by the underlying atmospheric background, the flux of the target is isolated in the focal plane of the telescope by the use of an array (center-filled hexagon) of optical fibers which acts as a diaphragm. The light from the telescope is incident on a slant mirror with the embedded bundle of optical fibers. Target and sky background photons are then transferred through the fibers to Avalanche Photodiode (APDs) single photon counters.

For polarization measurements an IR-blocking filter and a rotating polarization filter (RPF, Polaroid) are mounted into the incoming beam. The RPF covers the entire field of view and modulates the polarized component of stellar, nebular and sky background light twice over a full rotation. Typical rotation rates of the RPF are $3-5 \mathrm{~Hz}$. The RPF angular phase at any instant can be reconstructed from the signal of a Hall sensor switch that is triggered once per rotation and timed in the same way as a photon signal. The field around the fibers, visible in the slant mirror (typical size $2^{\prime \prime} \times 3^{\prime \prime}$ ), is imaged with a target acquisition camera. The polarizing filter modulates the incoming light effectively only over a wavelength range of about $470-750 \mathrm{~nm}$. Since the APD response ( $\mathrm{QE}>20 \%$ ) extends from about $450 \mathrm{~nm}$ to $970 \mathrm{~nm}$ and no wavelength information of the individual recorded events is available, it is necessary to block radiation outside the filter modulation range. Such photons, especially towards the near IR, are not modulated and would decrease the estimate for the degree of polarization. Therefore, we inserted an IR blocking filter that cuts the wavelength range at about $750 \mathrm{~nm}$. The OPTIMA detector is operated with two PCs and is autonomous except for the need to have a good telescope guiding system. 

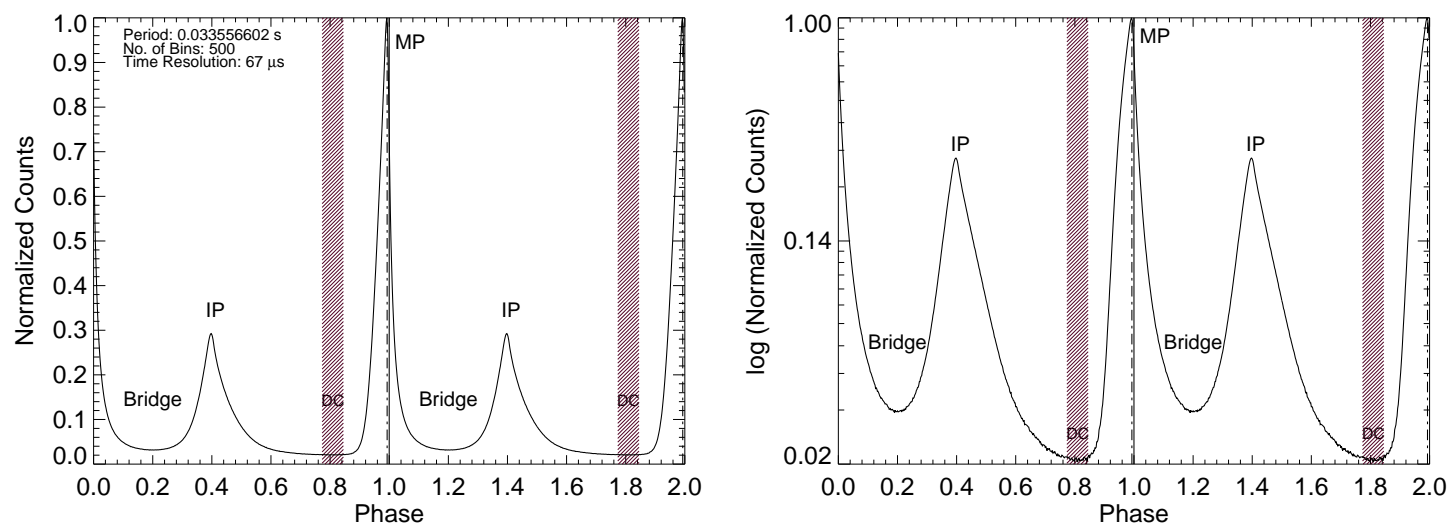

Figure 1: Left panel: the Crab pulsar light curve obtained from photons recorded by the OPTIMA central fiber APD at the Nordic Optical Telescope. The components of the light curve are indicated as follows: a main pulse MP, an inter pulse IP, a non-zero intensity level between the MP and IP, i.e. a bridge, as well as, a DC region. The DC component, in the phase interval $0.7729-0.8446$, was previously known as the 'offpulse' component. Right panel: the same as in the left panel but after nebula and background subtraction. To better show the DC intensity level it is plotted in a logarithmic scale. Two rotation periods are shown for clarity.

\section{Optical polarization characteristics of the Crab pulsar}

For each photon the phase angle of the RPF and the phase of the Crab pulsar are calculated. The latter involves the transfer of the topocentric photon times of arrival to the solar system barycenter, and the phase folding with the current pulsar ephemeris (Jodrell Bank Crab Pulsar Monthly Ephemeris). Hence, the pulsar signal is recovered by phase-coherent folding of sufficiently long observations at the pulsar's rotational frequency of about $29.8 \mathrm{~Hz}$ (Fig. 1).

The rotating polarization filter provides polarimetric data that represent a series of 'images' of an object taken through 180 sets of linear polarizers, when we bin the continuous rotation of the RPF into discrete one degree intervals. A single polarizer is not a $100 \%$ perfect polarizer, but its characteristics are well established, and this is essential for the chosen data analysis method. From an input data set of 180 independent intensities $I$ (measured in counts) and their errors $\left(\sigma_{I}=\sqrt{I}\right)$ corresponding to a set of observations through 180 identical but not perfect polarizers - we derive the Stokes parameters following the case of $n$ polarizers after Sparks \& Axon [3] for all seven fibers as a function of the Crab pulsar rotational phase. For the background (nebula and sky) subtraction we took the averaged Stokes parameters $\left(I_{D C}, Q_{D C}, U_{D C}\right)$ recorded in the ring fibers over the 'offpulse'. Pulsar optical polarization characteristics as a function of its rotational phase are calculated from the background subtracted Stokes parameters (Fig. 2).

Optical emission from the Crab pulsar is highly polarized, especially in the bridge and 'offpulse' phases. The position angle and the degree of linear polarization as functions of rotation phase show well determined properties. The position angle at the bridge and 'off-pulse' phases is constant. The polarization characteristics of both, the MP and the IP components, are quite similar. The polarization degree reaches a minimum at a phase close to the radio peak - the minimum is not aligned with the MP optical maximum. There is a well defined bump in the polarization degree 

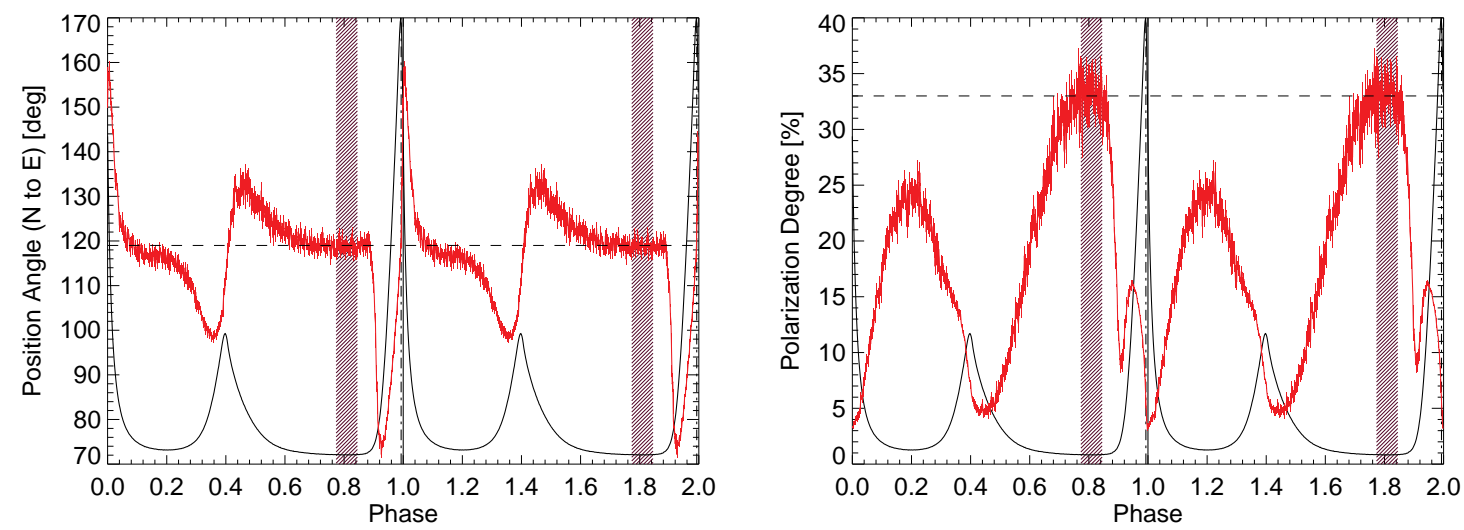

Figure 2: Linear optical polarization of the Crab pulsar: the position angle (PA, left panel) and the degree of polarization ( $\mathrm{PD}$, right panel) as a function of the pulsar rotational phase. Two rotation periods are shown for clarity. The PA changes are aligned with the MP maximum of the optical light (vertical dot-dashed line), but also with the zero phase, i.e. with the MP radio phase (vertical solid line). The PD minimum is reached at the radio phase (vertical solid line), and not at the phase of the optical maximum (vertical dot-dashed line). The optical pulse profile (solid line) and the DC phase range (dashed region in the phase range $0.78-0.84$ ) are indicated also. The DC component is constant, and has PA $\sim 119^{\circ}$ and $\mathrm{PD} \sim 33 \%$ (horizontal dashed lines). One sigma errors are show.

on the MP rising flank. There is an indication of such a bump also for the IP (especially after DC subtraction). The position angle swings through a large angle in both peaks. After subtraction of a constant DC component the angle swing is $130^{\circ}$ and $100^{\circ}$ for the MP and IP, respectively. The position angle slope changes dramatically at phases 0.993 (the MP maximum) and 1.0 ( $\equiv 0.0$, i.e. the radio peak). The trailing wing of the MP (phase range $0.0-0.03$ ) shows a linearly increasing degree of polarization. This feature turns into a bump shape after DC subtraction. There is a slight indication of the same behavior for the trailing wing of the IP. More details (including figures after DC subtraction), as well as, the discussion of our results in context of currently available theoretical pulsar models are given in [1].

\section{Polarization of the Crab neighborhood nebular background}

The Crab synchrotron nebula is a relativistic magnetized plasma that is powered by the spindown energy of the pulsar. It was the first recognized astronomical source of synchrotron radiation. The inner synchrotron nebula is a region consisting of jets, a torus of X-ray emission, small-scale variations in polarization and spectral index, and complexes of sharp wisps. A breakthrough in the optical studies of the structure of the Crab nebula was undertaken by using the Wide Field and Planetary Camera 2 (WFPC2) on board of the Hubble Space Telescope [Џ]. Hester et al. discovered a bright knot of visible emission located $0 . " 65$ to the south-east of the pulsar, along the axis of the system (Fig. 3). This inner knot, along with a second similarly sharp but fainter knot (hereafter outer knot) located at a distance of 3."8 from the pulsar, lies at an approximate position angle of $\sim 115^{\circ}$ east to north. Both knots are aligned with the X-ray and optical jet to the south-east of the pulsar and are elongated in the dimension roughly perpendicular to the jet direction, with lengths of 

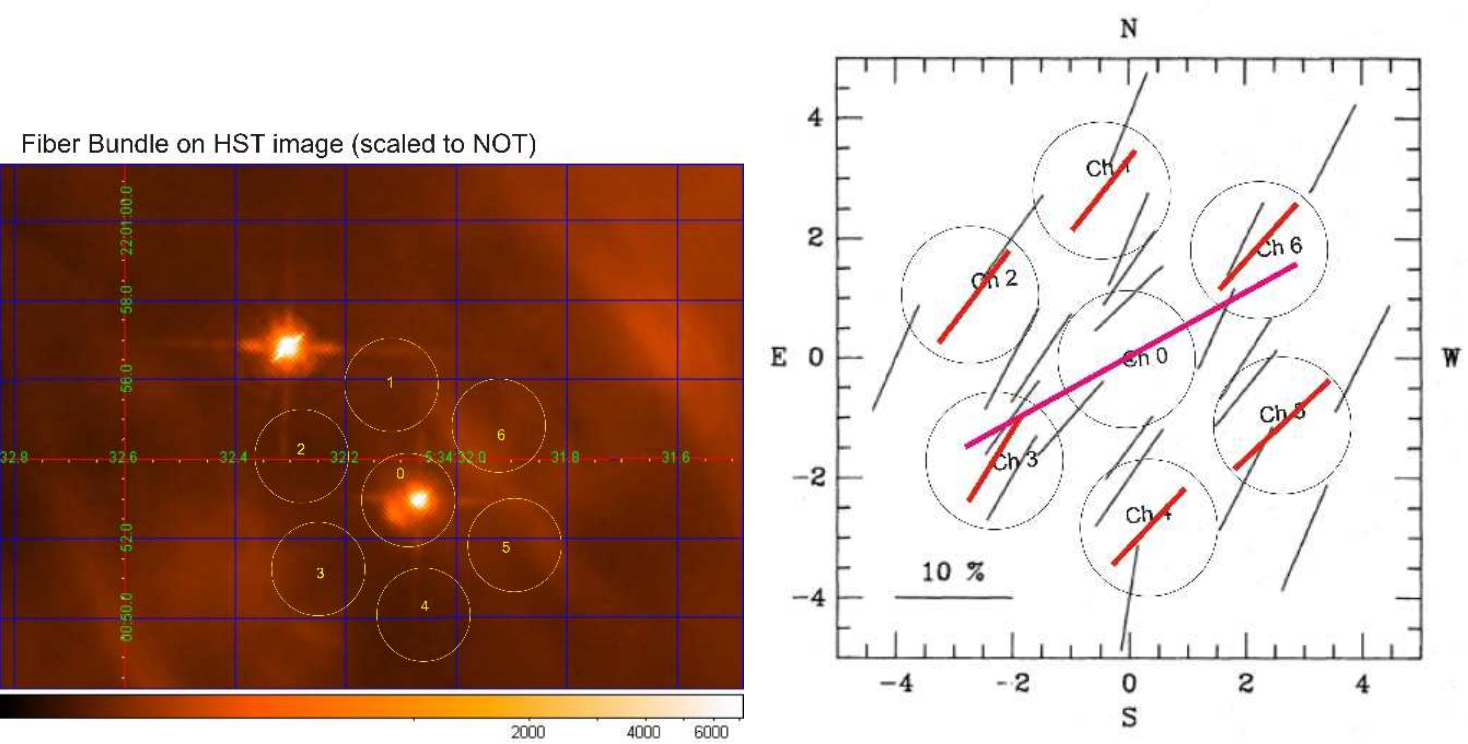

Figure 3: Left panel: an enlargement of the HST image of the inner Crab nebula (source R. Romani, priv. comm.). North is up; East is left. The pulsar is identified with the lower/right of the two stars near the geometric center of the nebula. A small arc-like feature (the inner knot) which is clearly resolved in the HST image is located 0." 65 to the SE of the pulsar. The OPTIMA fiber bundle, centered on the pulsar and scaled with the NOT focal plane scale, is over plotted. Right panel: polarization of the Crab nebula as measured by each single fiber at the minimum phase of the pulsar light curve. The axes are calibrated in arc seconds centered on the pulsar. The scaled OPTIMA fiber bundle is over plotted on the figure given in Smith et al. (1988). The aperture of a single fiber is 2 !' 35 . The pink line for central fiber and the red lines for the ring fibers represent our measurements with the rotating Polaroid filter. The black lines are the results that Smith et al. determined with a polarimeter based on a rotating half-wave plate and a Foster prism [5].

about a half arc second. The inner and outer knot appear to be present but not well resolved in the images of the Crab nebula previously taken by ground based telescopes. Left panel of Fig. 3 shows an enlargement of the co-added HST WFPC2 images of 12 observations of the Crab nebula (Roger Romani, priv. comm.). The OPTIMA fiber bundle centered on the pulsar and scaled with the NOT focal plane scale is over plotted. The target fiber is central to a hexagonal bundle of identical fibers which measure the sky background and, in the case of the Crab nebula, the close environment of the pulsar. It is clear that we are not able to resolve the Crab pulsar from the inner knot within the central fiber.

To obtain the polarization characteristics of the pulsar neighborhood we assumed that within the DC phase range the contribution of the pulsar emission to the ring fibers is minimal. Therefore, for background (nebula) calculations we consider only light coming within the 'off-pulse' phase range, i.e. $7 \%$ of the whole rotational cycle of the Crab pulsar. Obtained polarization degree and position angles for each of the single OPTIMA apertures are shown in the Fig. B, right panel. We compared our results with previous ones by over plotting them on the polarization sky map of the very close neighborhood presented by Smith et al. [5]. By averaging the Stokes parameters over all background channels we get $\mathrm{PD}=9.71 \% \pm 0.08 \%$ and $\mathrm{PA}=139.8^{\circ} \pm 0.2^{\circ}$ for the region surrounding the pulsar. Close to the pulsar the nebular polarization is quite uniform $(\sim 9-11 \%)$ 
but the position angles change steadily with radial distance. Two to three arc seconds from the pulsar the mean value is around $140^{\circ}$, but beyond five arc seconds the position angle exceeds $155^{\circ}$ and it is very position-dependent [6].

\section{Acknowledgments}

AS acknowledges support from the EU grant MTKD-CT-2006 039965.

\section{References}

[1] A. Słowikowska et al., Optical polarisation of the Crab pulsar: precision measurements and comparison to the radio emission, Accepted for publication in MNRAS (2009) [arXiv:0901.4559v2].

[2] G. Kanbach et al., High Time Resolution Astrophysics, Springer Netherlands, 2008.

[3] W. B. Sparks \& D. J. Axon, Panoramic Polarimetry Data Analysis, PASP 111 (1999) pp.1298-1315.

[4] J. J. Hester et al., WFPC2 Studies of the Crab Nebula. I. HST and ROSAT Imaging of the Synchrotron Nebula, ApJ 448 (1995) pp.240-263.

[5] F. G. Smith et al., The optical polarization of the Crab Pulsar, MNRAS 233 (1988) pp.305-319.

[6] I. S. Mc Lean et al., High-resolution polarization images of Crab Nebula with a charge-coupled device camera, Nature 304 (1983) pp.243-246. 